\title{
EFFECT OF HIGH TEMPERATURE ON MEIOSIS IN LOLIUM: NUCLEOLAR INACTIVATION
}

\author{
H. K. JAIN \\ Department of Agricultural Botany, Aberystwyth *
}

Received 8.vi.56

\section{INTRODUCTION}

Following their emergence from the resting stage, chromosomes begin to contract lengthwise and continue to do so throughout prophase, towards the end of which their short and cylindrical form is in marked contrast to the threadlike appearance earlier. The process of chromosome contraction, apart from its role in making them mechanically efficient, is considered important for the changes that it signifies in their chemical organisation, because of which the term "condensation" is generally used to describe it. Numerous studies of chromosome structure during various stages of the division cycle have helped to establish the fact that their linear contraction depends upon the assumption of a spiral form and that uncoiling at telophase restores the early prophase condition.

The degree of coiling of different chromosomes in the same complement is usually equal and constant, a fact which is responsible for their uniform thickness and constant lengths. Moreover the process of condensation is usually synchronised for all members of the complement so that at any particular stage in the nuclear cycle, they are found to be in similar states. Many exceptions, however, are known where particular chromosomes or parts of them do not keep step with the rest of the complement in their condensation cycle. These chromosomes or regions because of their differential behaviour were described as heterochromatic by Heitz (I93I) who was the first to study them in great detail. The best known examples are the sex chromosomes in Drosophila and other insects, but autosomal regions in many organisms are found to be similarly affected (Darlington, 1937).

The investigations reported here concern experimentally induced differences in the degree of condensation of chromosomes which are members of the same complement and whose uniform and synchronised condensation is normally characteristic.

Experimentally induced changes of this nature bring to notice new characteristics of chromosomes, which may be of more than a limited interest. With the advances made in nuclear chemistry in recent years it has been possible to interpret purely morphological observations on chromosomes in terms of cytochemical changes. As well as contributing to a deeper understanding of chromosome

* Present address : Botany Division, Indian Agricultural Research Unit, New Delhi I 2 
behaviour, such correlated studies provide means of testing the chemical evidence which in the absence of absolutely reliable techniques is not always decisive. If this evidence is to be accepted, it should be possible to reconcile it with the observed behaviour of chromosomes. In a normal cell division, however, the co-ordinated changes in the nucleus obscure the individuality of chromosomes and their observed behaviour is not very revealing. Therefore, the need for inducing changes, which considerably increase the scope for the interpretation of cytochemical evidence.

\section{MATERIAL AND METHOD}

Population plants of perennial rye grass (Lolium perenne L.) which formed part of a collection for genetic studies provided the material. These have been clonally multiplied for a number of years and have given standardised controls. The treatment applied to induce the cytological changes, consisted of subjecting the potted plants to high temperatures before and during part of meiosis. The treatments were given in electrically heated and thermostatically controlled chambers.

Different temperature treatments have been tried, the successful ones being durations of $14,3^{8}$ and $5^{8}$ hours at $35^{\circ} \pm 2^{\circ} \mathrm{C}$. followed by immediate fixation. Both fixation and storage were done in Carnoy to which a few drops of aqueous ferric chloride solution had been added. Staining was done mostly in acetocarmine, the other stain used being Feulgen which gave similar but not equally good results.

\section{RESULTS}

(i) Mixed nuclei

Meiosis in the perennial rye grass clones used is quite regular under normal conditions. All its I4 chromosomes, which is the diploid number, are found to be synchronised throughout the nuclear cycle, especially in respect of their prophase condensation which appears to be uniform for all members of the complement as also for all parts of individual chromosomes (plate I, fig. I). Seven bivalents are regularly formed and these are co-ordinated in their congression and co-orientation on the metaphase plate. The nucleolus is equally regular in its formation. Study of the nucleus at earlier stages show that two nucleoli are organised in close association with two chromosomes and these fuse to form the single nucleolus found at later stages of prophase (plate I, fig. 2). It disappears shortly before the spindle is organised.

Following the three temperature treatments described earlier, the regular behaviour of chromosomes during meiotic prophase is visibly upset. The experimentally induced irregularities in the nucleus are as follows :

A very obvious change is manifested in the operation of a dual system in the prophase development of various members of the complement. The co-ordinated condensation of chromosomes in two different non-synchronised groups is particularly conspicuous, those in a normal group being far more contracted and deeply stained than the ones in the other retarded group. The differences, however, 
are not always equally pronounced. In extreme cases chromosomes showing maximal condensation may be present along with others which have hardly undergone any spiralisation at all. In less extreme cases, diakinesis bivalents and those approaching diplotone may be found in the same nucleus (plate II, $c f$. figs. I and 6).

\section{(ii) Frequencies}

The abnormal behaviour in respect of condensation is evidently not characteristic of particular chromosomes in the complement since the number of affected chromosomes varies, as shown in table below, from cell to cell. Six fully condensed and one relatively uncondensed bivalent or two unspiralised chromosomes may be present in one pollen mother cell, while in another, the same number of chromosomes may be found in a reversed state of condensation (plate II, $c f$. figs. I and 9).

The following table compiled from observations on a random sample of 66 cells from two sister anthers shows the frequency of pollen mother cells with all their chromosomes normally condensed and others having varying numbers of condensed and relatively uncondensed chromosomes.

TABLE

Showing the variation in frequencies of $P M C$ with retarded bivalents following treatment for $5^{8}$ hours at $35^{\circ} \pm 2^{\circ} \mathrm{C}$.

\begin{tabular}{|l|c|c|c|c|c|c|c|}
\hline Chromosome complement & \multicolumn{7}{|c|}{$2 n=14=7$ II } \\
\hline $\begin{array}{c}\text { Number of normal bivalents (with } \\
\text { attached nucleoli) }\end{array}$ & I & 2 & 3 & 4 & 5 & 6 & 7 \\
\hline $\begin{array}{c}\text { Frequency of pollen mother cells } \\
\text { (total 66) }\end{array}$ & 13 & 7 & 4 & 5 & 5 & 4 & 28 \\
\hline
\end{tabular}

N.B. - No normal cells with single normally organised nucleoli were found in this sample, although they occur perhaps in I per cent. of PMC.

The other temperature treatments give similar results with regard to the frequency of PMC's with varying numbers of condensed and uncondensed chromosomes.

Non-synchronised appearance of chromosomes such as described here may obviously result from two entirely different causes. Precocious development of some of the chromosomes or their failure to condense at the normal rate, would both produce the same phenotypic effect in the nucleus. The following observations clearly suggest failure of condensation and rule out the possibility of precocity in this particular instance.

(I) If the apparently retarded chromosomes were condensing at a normal rate, they should be able to reach their usual metaphase degree of condensation and catch up with the supposedly precocious 
chromosomes towards the end of prophase. This they never do, thus indicating a failure on their part, which in some cases is very considerable, as already described.

(2) The fully condensed chromosomes are invariably present as bivalents and not as univalents. This would not be likely if the chromosomes concerned showed precocious condensation before pachytene, a fact which would be expected to interfere with their pairing and chiasma formation. Nor is there any evidence for a particularly rapid condensation of the chromosomes after pachytene; the various stages leading to metaphase condition can often be seen.

The fact that the normally condensed chromosomes occur as bivalents and not as univalents is significant in more than one respect. It also shows that the homologous chromosomes respond to the temperature treatment in the same manner-both showing normal condensation or its failure.

It was pointed out earlier that the failure of condensation in the affected chromosomes may be complete or partial. The extent of this failure, however, varies.

In pollen mother cells in which a large number of chromosomes, as many as 5-6 bivalents, show normal condensation, the remaining few chromosomes appear almost completely unspiralised and hardly stain at all. It is usually obvious that the affected chromosomes have advanced little beyond their interphase condition. Occasionally, however, they enter prophase and the homologues may even pair and form chiasmata but their stainability is never appreciably improved (plate II, figs. I-4). Or the contrary in those cells in which only one or two fully condensed bivalents make their appearance, the remaining members of the complement also show a fair degree of condensation, their stainability is particularly improved and they do not present a woolly appearance so characteristic in the former case (plate II, figs. 6-12). These chromosomes in many cases, are able to pair and form chiasmata, although the latter formed bivalents never quite catch up with others in their degree of condensation. An intermediate behaviour with regard to their stainability and spiralisation is shown by the affected chromosomes in those cells in which three or four fully condensed bivalents are present (plate II, fig. 5).

Thus the degree of retarded chromosomes is related to and determined by the number of normal bivalents in each nucleus. Apart from this, the behaviour of the retarded chromosomes is more variable when only one or two normal bivalents are present. In such cells they may appear as retarded bivalents with regular or rather fuzzy outlines (plate II, $c f$. figs. 6 and 9). They may show failure of pairing (plate II, fig. Io), or they may be clumped, a condition with or without stickiness (plate II, $c f$. figs. 7 and I I).

It may also be emphasised that normal and retarded chromosomes are found to be randomly distributed in the nucleus. There is no suggestion that they occur in groups. 
Finally it should be pointed out that not all pollen mother cells in the treated material include retarded chromosomes, but as many as 60 per cent. may be affected in particular anthers. The number varies considerably, both with different treatments and also within the same treatment. Only certain of the genetically different clones respond to the temperature treatment and in these only some flowers in some of the spikes.

\section{(iii) Nucleoli}

Abnormal behaviour of the chromosomes is not the only change induced in the nucleus. Other changes, which appear to be correlated with them, concern the nucleolus. The retardation of various chromosomes during meiotic prophase is always accompanied by abnormal nucleolar activity. The main irregularity is that no single nucleolus has usually been formed in the preceding resting stage. Instead, several nucleolar bodies are formed, usually as many as the number of normally condensed chromosomes with which they are found to be closely associated (plate II, figs. I, 3, 5-I2). Their number thus usually varies from I-6, depending upon the number of normally condensed bivalents. Sometimes, however, more than one nucleolar body may be associated with such chromosomes or, alternatively, more than one bivalent may be associated with a single nucleolus. In still other cases, the nucleolar bodies may be found detached from the chromosomes. But their proximity to normal chromosomes and not to the retarded ones can always be observed and indicates association at earlier stages.

Thus the nucleolar bodies are found in association with the condensed chromosomes only. Chromosomes which show failure of condensation never have any nucleolar material attached to them. It is also very obvious that the presence or absence of nucleolar material on the chromosomes is not determined by their particular state of condensation. The association with some chromosomes and not with others can also be seen at earlier stages of divisio when all of them are present in the same relatively uncondensed state (plate I, fig. 3).

There seems little doubt that with the failure of the nucleolar organiser, individual chromosomes can have their own nucleolar material in many of the cells; the nucleolar bodies thus formed are best described as adventitious (plate I, fig. 4). In many other cells, however, a number of chromosomes fail to have attached nucleoli and these are the ones which show failure of normal condensation.

The number of abnormal chromosomes varies greatly, as already pointed out, but cells with one or two normal bivalents occur with the greatest frequency. These frequencies suggest that the chromosomes differ in their capacity to form the nucleoli after the organiser has been inactivated. The bivalents, however, cannot be distinguished from one another and the suggestion therefore cannot be confirmed. 

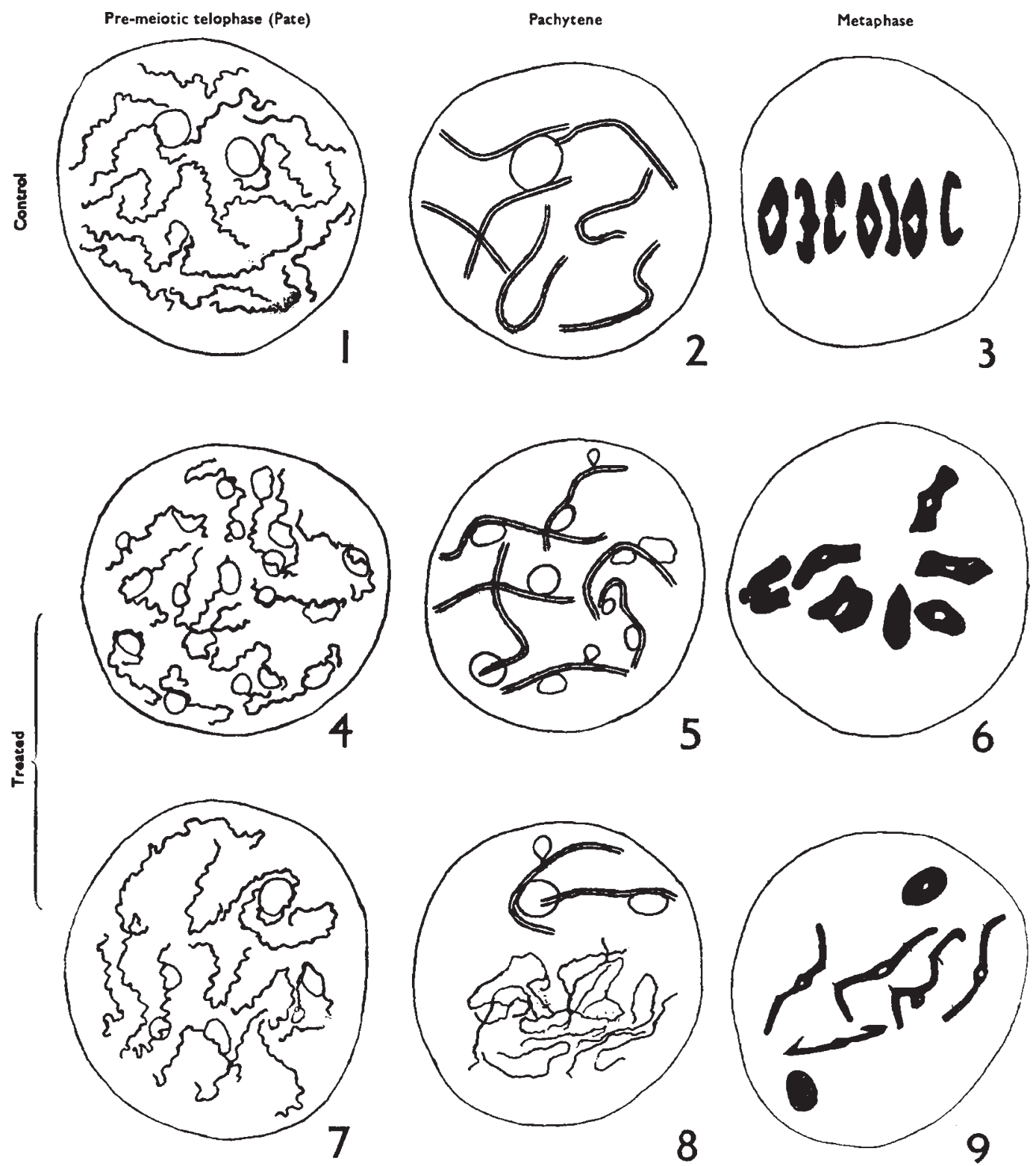

5
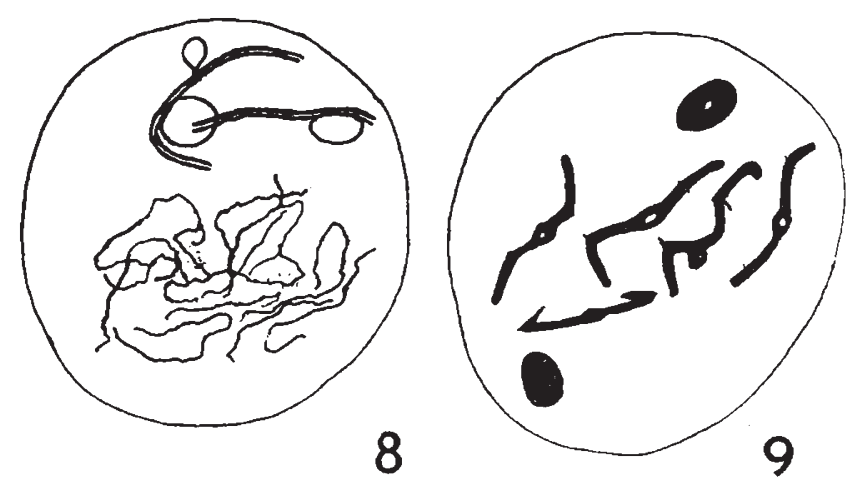

Fig. 1. Normal and abnormal nucleolar formation at pre-meiotic telophase and the subsequent behaviour of chromosomes in the control (figs. 1-3) and treated (figs. 4-9) material. 1. Normal formation of two nucleoli by the two organising chromosomes. 2. The seven bivalents synchronised in their development. 3. The normal metaphase arrangement. 4. Each of the fourteen chromosomes adventitiously forming nucleolar bodies. 5. The seven bivalents with associated nucleolar material and all synchronised as in 2. 6. Irregular metaphase arrangement; partial congression and failure of coorientation. 7. Two pairs of chromosomes taking part in nucleolar synthesis. 8. Nucleolar-forming chromosomes showing pachytene pairing, others beginning to emerge from resting stage. 9. The two normal bivalents failing to congress, the retarded ones failing to co-orientate. 


\section{(iv) Metaphase}

The non-synchronised development of chromosomes and the formation of adventitious nucleoli culminates in a metaphase condition which is highly irregular. The spindle in many of the PMG fails to be organised. In others the congression and co-orientation of bivalents on the metaphase plate are affected. When all the 7 bivalents in a cell are fully condensed, these may congress on the plate but their centromeres often fail to co-orientate showing instead an apparent lack of any orientation or a condition similar to auto ${ }^{\perp}$ orientation of mitotic chromosomes. When both normal and retarded bivalents are present, the condensed ones may fail to show the metaphase arrangement and remain randomly distributed in the cell. The retarded ones, however, are often able to congress although their centromeres do not coorientate.

The chromosomes following the irregular metaphase form one or more restitution nuclei, which apparently degenerate and no viable pollen grains are ever formed.

In the above account we have seen how the differential behaviour of chromosomes is to be associated with abnormal nucleolar activity. The diagram gives a general illustration of these and other results (fig. I).

\section{DISCUSSION AND CONCLUSIONS}

For an understanding of the experimentally induced changes, two explanations are needed. First, the partial or complete failure of condensation in some members of the complement, when others condense quite normally, has to be explained. Secondly, it has to be determined whether this failure is in any way related to abnormal nucleolar formation, which is a characteristic feature of those nuclei whose chromosomes show such differences.

The suggestion that the irregular behaviour of chromosomes may be directly related to abnormal nucleolar formation is difficult to avoid. The nucleolar material is found to be associated with some of the chromosomes and not with others during earliest prophase, when all of them are present in a relatively uncondensed state. Similar differences in the association of the nucleolar material are still present during later stages of prophase, but the chromosomes are no longer found to be synchronised in their degree of condensation. Only the ones with nucleolar material attached appear normally condensed. The usual reduction in the size of the nucleolar bodies towards the end of prophase can also be seen. In view of these observations it is tempting to suppose that the nucleolar material may be responsible in some way for the condensation of chromosomes to which it is seen to be attached, while the lack of such association in others may account for their poor condensation. This is particularly so in view of the numerous reports in cytological literature which specifically attribute this role to the nucleolus substance. 
Since nucleoli in most dividing cells disappear at the same time as chromosomes reach their maximum contraction and reappear in telophase with the reversal of prophase changes, many workers have assumed that the nucleolar material was incorporated into the chromosomes during their condensation. McClintock (1934) was one of the first to put forward this idea.

Darlington (1947) referred to the fact that when there is no organiser or nucleolus at all in an organism, there is always heterochromatin, and when there is no heterochromatin there is always a nucleolus. He took the broader view that the nucleolus and the charged heterochromatin evidently provide alternative or supplementary sources of the nucleic acid used in the charging of the prophase chromosomes. The possibility of the absorption of the nucleolar material into the substance of chromosomes has been suggested by White (195I) who pointed out that most authors have tended to regard the nucleoli as a temporary storage reservoir of material which is transferred to the chromosomes before metaphase.

If the suggestions made by McClintock and others are to be accepted, the induced differences in the condensation of different chromosomes in relation to nucleolar changes could have a simple explanation. With the inactivation of the nucleolar organiser as a result of temperature treatment, chromosomes synthesise individually the nucleolar material which remains associated with them, in the same way as in maize under conditions of a different nature. McClintock (1934) has shown that in pollen grains with defective nuclei-ones without the organising chromosome present-nucleoluslike droplets appear over the chromosomes generally. In others, particular genotypic changes may suppress the organising capacity of the nucleolar chromosome and induce the formation of adventitious nucleoli. However, many of the affected PMC in Lolium differ from the defective maize pollen grains in one important respect. In these following the inactivation of the organiser, not all members of the complement form the adventitious nucleoli. Some of the chromosomes fail to do so and these might be expected to remain uncondensed during prophase on the basis of McClintock's hypothesis. The dispersed nucleoli, unlike the normally organised ones, it might be held, do not contribute to the metabolism of all members of the complement but only of those to which they are attached.

A number of considerations, however, make it difficult to accept this simple explanation.

The concept of the transfer of nucleolar material to chromosomes and their consequent condensation, though very natural in view of their apparently related behaviour, is rendered unlikely in view of all the evidence. The Feulgen negative character of the nucleolus is well known and is readily demonstrated in the present material. Also the presence of ribose nucleic acid (RNA) in it has been demonstrated by a large number of workers. The discovery that RNA is 
present in the chromosomes and more recently its reported increase in mitotic chromosomes compared with the interphase nucleus, may support the hypothesis of nucleolar contribution to the chromosomes. However, Jacobson and Webb (1952) who among others reported the increase, also pointed out that the ribonucleo-proteins appear on the chromosomes during the prophase while the nucleolus is still present and of normal size. In their view there is no evidence that the entire ribonucleo-protein of the chromosome is derived from the nucleolus. Moreover, the presence of RNA in the chromosomes has not obscured the fact that the nucleic acid content of the chromosomes is predominantly the Desoxyribose type. The possibility that the nucleolar RNA may be converted into Desoxyribose nucleic acid (DNA) of the chromosomes would be entirely opposed to the current views on the subject. Brachet (1947) who among others suggested that RNA may be a precursor of DNA, has since stated that the hypothesis of conversion seems doubtful. Mirsky (I95I) is more definite and states that there is no evidence at present that DNA is formed from RNA or vice versa. The possibility that DNA may be controlling RNA synthesis has, however, been suggested by Rich and Watson (1954).

There are other good arguments against the views of McClintock and others. The quantitative instability of the ribonucleo-proteins of the nucleolus and of the cells in general, emphasised by several authors (Swift, I953; Alfert, I954; Mirsky, I951) makes it difficult to relate the nucleolar material with a regular process like chromosome condensation. During the course of these and other cytological investigations on rye grass, not only great variations in nucleolar size have been observed, but it has been noted occasionally that metaphase chromosomes after the disappearance of nucleolar material may be condensed to a lesser degree than the diakinesis chromosomes in a neighbouring cell in which the nucleolus is still present. An alternative explanation for the disappearance of the nucleolus shortly before metaphase has been provided by the work of Caspersson and his collaborators (1950) who believe that the nucleolus is associated with cytoplasmic protein synthesis and its disappearance merely marks the end of that activity.

The above discussion shows that the induced differences in the degree of condensation of different chromosomes in the complement are not easily explained as due to the association of nucleolar material with a restricted number of chromosomes only. In view of this the first question may now be considered afresh in the light of other relevant evidence concerning chromosome condensation.

Corresponding with their cycle of spiralisation and despiralisation during mitosis, chromosomes are involved in another cycle of changes in their stainability and the amount of ultraviolet-absorbing materials concentrated on them. While the despiralised interphase chromosomes hardly stain at all, the maximally contracted metaphase chromosomes 
do so very intensely. The prophase condensation of the chromosome threads has been attributed to the material, the so-called matrix which is responsible for their increased stainability (Wilson and Coleman, I952; Cleveland, 1949). As for the nature of this substance, cytochemical methods and ultraviolet absorption techniques show that desoxyribo-nucleohistones are concerned (Jacobson and Webb, r952a; Alfert, 1954). Apart from the histones which Mirsky and Ris (I95 I) suppose to be quantitatively correlated with DNA, the protein content of nuclei decreases during division cycle. Discussing this, Pollister (1952) points out that the increase in DNA concentration as the chromatin condenses into compact chromosomes is not accompanied by corresponding protein condensation and that therefore the actual protein mass must be very much less in the metaphase chromosomes.

The evidence thus leaves little doubt that the prophase condensation of chromosomes is brought about by the concentration of the DNA-histones on the interphase threads. Darlington (1955) has described DNA as the essential agent in spiralisation and as evidence points to the fact that in certain polyploid plants and in the X-chromosome of hamster and other mammals, spiralisation fails with extreme starvation. As to the mechanism of the process Darlington has stressed the importance of residual protein. Referring to the work of Mirsky and Ris, he explains that it is the combination of polymerised DNA and residual protein which maintains the fibrous structure and thus must determine the spiralisation of chromosomes. Caspersson (1950) has suggested a specific mechanism for the process. According to him, the chromosome contracts with spiralisation: a phenomenon which might be caused by the small differences in the spacing of the nucleotides and polypeptide chains.

The failure of some of the chromosomes to condense must therefore be considered in terms of changes in their DNA content. The explanation needed is how some of the chromosomes come to have less than the normal amount of DNA that ensures complete spiralisation in others. The possibility that the failure of chromosome condensation may merely indicate a failure of the DNA to concentrate on them rather than its actual shortage has been considered. It does not, however, accord with the observed behaviour of the affected chromosomes. It has already been pointed out that the extent of failure of condensation in the retarded chromosomes is determined by the number of normal chromosomes present in the nucleus: the greater the number of such chromosomes, more complete is the failure of condensation in others. This relationship cannot be explained excep! on the basis of quantitative differences, which suggest competition between the normal and affected members of the complement.

The changes in the DNA content of different chromosomes evidently arise during interphase, for it has been noticed in many cases that the affected chromosomes barely emerge from that state (plate II, fig. I). Moreover, this is also the stage according to recent 
studies of mitosis if not of meiosis when synthesis of fresh DNA starts during the course of mitosis (Swift, r953; Alfert, 1954; Walker and Yates, 1952). In meiosis synthetic activity it would appear is continued during prophase or, as Ansley (1954) has reported in the centipede, is confined to leptotene and zygotene.

In order to understand how these quantitative changes arise, it is necessary to refer to the distribution of DNA content of the interphase nucleus in relation to chromosome threads. Evidence on this point is not conclusive, and it is not definitely known whether the DNA leaves the chromosomes during this stage or merely becomes less concentrated.

If the DNA is detached from the chromosomes and dispersed in the nuclear sap, the possibility of a competition between various chromosomes towards the end of interphase is not difficult to visualise. Also if the synthesis of DNA has not been complete, some chromosomes may be more successful than others purely on a chance basis. The evidence, however, is strongly against this view. Since the normal and retarded chromosomes are found to be randomly distributed, differences in their degree of condensation cannot be explained by postulating the existence of a nucleoplasmic gradient in the nucleus. Moreover, there is evidence that successful condensation, though not a function of particular chromosomes, is determined by factors intrinsic to the chromosomes rather than by circumstances outside their control : the homologous chromosomes always respond to the temperature treatment in the same manner-both showing normal condensation or its failure. This would certainly not be the case if successful condensation or its failure is determined on a random basis.

A more generally accepted view about the state of chromosomes in the interphase nucleus is that the chromatin material does not leave the chromosome threads at all, it merely becomes loosely attached. The swollen DNA histone component of the chromosomes is then described as existing in an extended state (Ris and Mirsky, 1949; Pollister, 1952). Following fresh synthesis, DNA begins to concentrate on the chromosome threads once again, when they make their prophase appearance. On this view, shortage in the DNA content of a number of chromosomes will directly result, if they failed to take part in the synthesis of fresh DNA. In other words, the reduced DNA content of a number of chromosomes is simply accounted for by their reduced synthetic activity for this substance. This is an interesting possibility in view of the evidence that the normally and poorly condensing chromosomes are characterised by differing synthesising capacities. This refers, of course, to their synthesis of nucleolar material.

It has already been described that with the inactivation of the nucleolar organiser, not all the chromosomes in an affected cell may synthesise the nucleolar material. It may be that the chromosomes show similar differences with regard to DNA synthesis. The observation that only the nucleolus-forming chromosomes show normal 
condensation may be interpreted as indicating that the capacities for nucleolar and DNA synthesis are correlated.

The reduced synthetic activity of the chromosomes may help to explain their failure of condensation. But the extent of this failure varies and is determined by the number of normally condensed chromosomes present in the nucleus. How do the normally condensed chromosomes exercise this control ? It would appear that the chromosomes with reduced activity have to compete with the normal ones for a limited amount of precursor material. In that case, as the number of normals increases, the amount available to the affected ones will be proportionately decreased.

An attempt has been made in the above discussion to interpret the essential features of the observations described earlier. The suggestion regarding the correlated capacities for nucleolar and DNA synthesis, emerging from this, could be considered further if only briefly. It may be that the two syntheses in the chromosomes are under a common control. This finds support in the observations made by Caspersson (1950) and Schultz (1947), based on their cytological studies of salivary gland chromosomes. To quote Schultz's own words, "The same region which synthesises blocks of desoxynucleoproteins in the chromosome has to do with ribonucleo-protein synthesis in nucleolus and cytoplasm and with different protein component of the chromosomes". This region in their view is the heterochromatin.

A number of considerations, however, indicate that the role of heterochromatin in this connection is not to be overstressed. Darlington (1947) has pointed out that many organisms lack both nucleolar organiser and heterochromatin, but the nucleoli are still formed. Moreover, when both are present, the organiser may lie in the heterochromatin or outside it. The observations of McClintock discussed earlier show that the function of heterochromatic nucleolar regions in maize is to organise material formed elsewhere in the chromosomes. In the absence of the organiser, the nucleolar material appears on all the chromosomes. That this is also what happens in rye grass when the organiser is inactivated has already been described in detail. These observations thus indicate that the role of euchromatic regions in synthesis cannot be ruled out.

\section{SUMMARY}

I. In certain clones of Lolium perenne, high temperature treatment at certain stages which include the telophase before meiosis, inactivates the nucleolar organiser in nearly all PMC. It disperses the materials so that nucleoli are formed by I-7 bivalents ; the partner chromosomes always act in the same manner.

2. When less than 7 bivalents form nucleoli, the non-nucleolar chromosomes are retarded in their DNA-reproduction and prophase condensation. The fewer bivalents are retarded, the more they are 

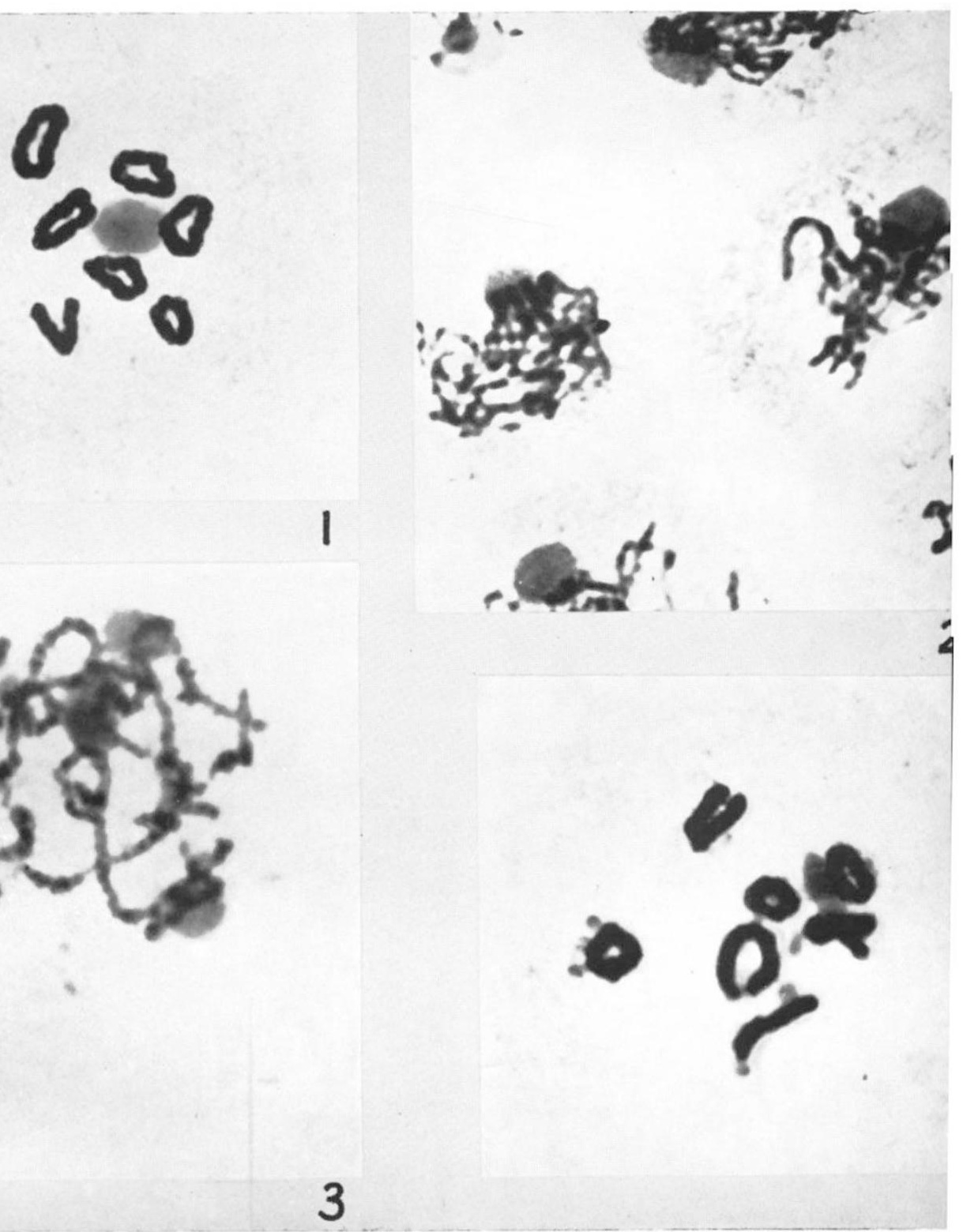

\section{Plate I}

FIG. I.-PMG showing the uniform condensation of all the seven bivalents at diakinesis in the control material. A single nucleolus can also be seen. $\times 2075$.

Fig. 2.-A patch of normal cells. A single nucleolus is seen in each of the prophase nuclei. $\times 1600$.

Fig. 3.-An affected cell at early prophase showing the nucleolar material associated with some of the chromosomes and not with the others, before condensation has occurred. Eight nucleolar bodies differing in size can be recognised. $5^{8}$ hours, $35 \pm 2^{\circ} \mathrm{C} . \quad \times 2075$.

Fig. 4.-Diakinesis in an affected cell. Each of the seven bivalents has nucleolar bodies on it and all appear normally condensed. $3^{8}$ hours, $35 \pm 2^{\circ} \mathrm{C} . \quad \times 2075$. 


\section{Plate II}

Diakinesis stages of the normal chromosomes in PMC showing the effects of treatment.

Fig. I.-Six normally condensed bivalents and two remaining chromosomes which have hardly emerged from their interphase condition can be seen. The nucleolar material is seen on all the normally condensed chromosomes. $5^{8}$ hours, $35 \pm 2^{\circ} \mathrm{C}$. $\times 1925$.

Fig. 2.-Five normal bivalents and two others which are underspiralised and show reduced stainability, can be seen. $5^{8}$ hours, $35^{\circ} \pm 2^{\circ} \mathrm{C}$. $\times 2525$.

Fig. 5.-Three fully condensed bivalents each associated with a nucleolar body. The affected chromosomes showing failure of condensation and reduced stainability. $3^{8}$ hours, $35 \pm 2^{\circ} \mathrm{C} . \quad \times 2525$.

Fig. 6.-Two normally condensed bivalents, both attached to a single nucleolus. The remaining chromosomes correspond to a diplotene stage. Rod bivalent at 2 o'clock. $3^{8}$ hours, $35 \pm 2^{\circ} \mathrm{C} . \quad \times 2075$.

Fig. 9.-One normally condensed bivalent with a reduced nucleolus. The partially condensed chromosomes appear as in a diplotene nucleus. $5^{8}$ hours, $35 \pm 2^{\circ} \mathrm{C}$. $\times 1925$.

Fig. Io.-One fully condensed bivalent with the nucleolus attached to it. The remaining chromosomes almost normally condensed but show failure of pairing. $3^{8}$ hours, $35 \pm 2^{\circ}$ C. $\times 2075$. 


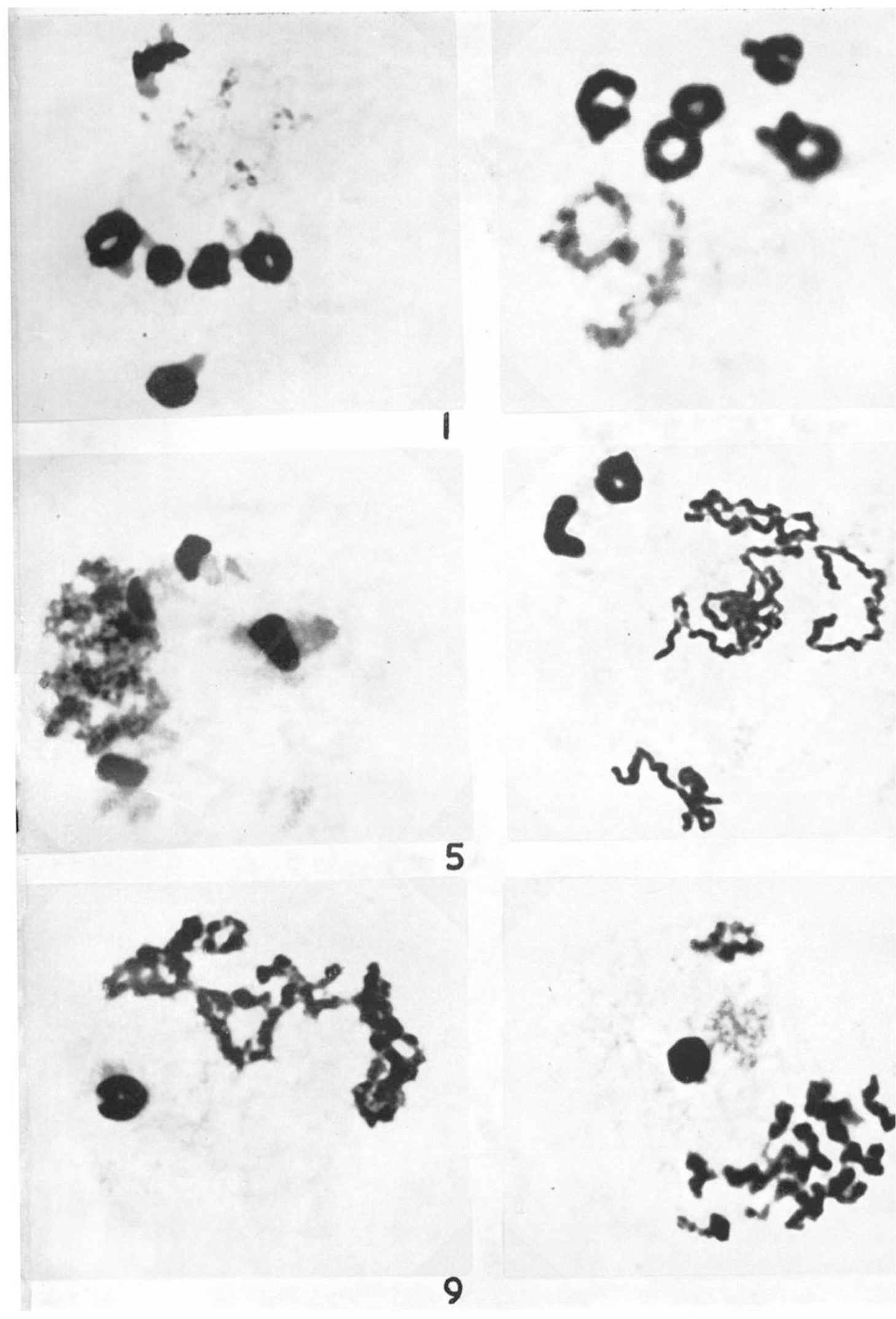


Diakinesis stages of the normal chromosomes in PMG showing the effects of treatment.

FIG. 3.- Six normally condensed bivalents having nucleolar material on them. The remaining two failing in their prophase development. $5^{8}$ hours, $35 \pm 2^{\circ} \mathrm{C}$. $\times 2525$.

Fig. 4.-Five normally condensed bivalents and the affected chromosomes showing somewhat improved stainability. $5^{8}$ hours, $35 \pm 2^{\circ} \mathrm{C} . \quad \times 1925$.

FIG. 7.-Two fully condensed bivalents with prominent nucleolar bodies. The remaining chromosomes clumped and showing good stainability. $5^{8}$ hours, $35 \pm 2^{\circ} \mathrm{C}$. $\times 1925$.

Fig. 8.- Two normally condensed bivalents, each with a nucleolar body: The affected chromosomes showing failure of normal condensation but their stainability is not much reduced. 58 hours, $35 \pm 2^{\circ} \mathrm{C}$. $\times 1925$.

Fig. I I, - One fully condensed bivalent with a large nucleolus attached to it. The remaining chromosomes showing stickiness. 14 hours, $35 \pm 2^{\circ} \mathrm{G} . \quad \times 2525$.

Fig. I 2.- One fully condensed bivalent with the nucleolus attached to it can be seen. The remaining chromosomes show good stainability and appear clumped. 14 hours, $35 \pm 2^{\circ}$ C. $\times 1925$. 


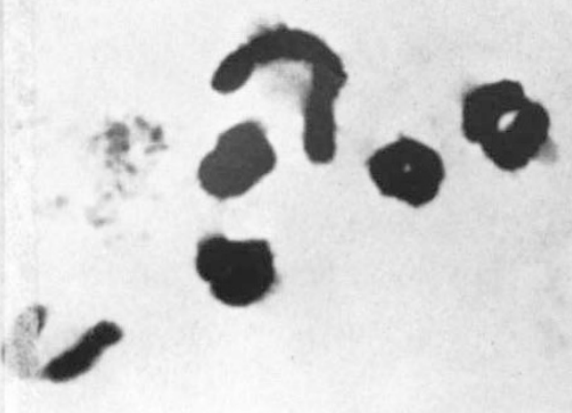

3
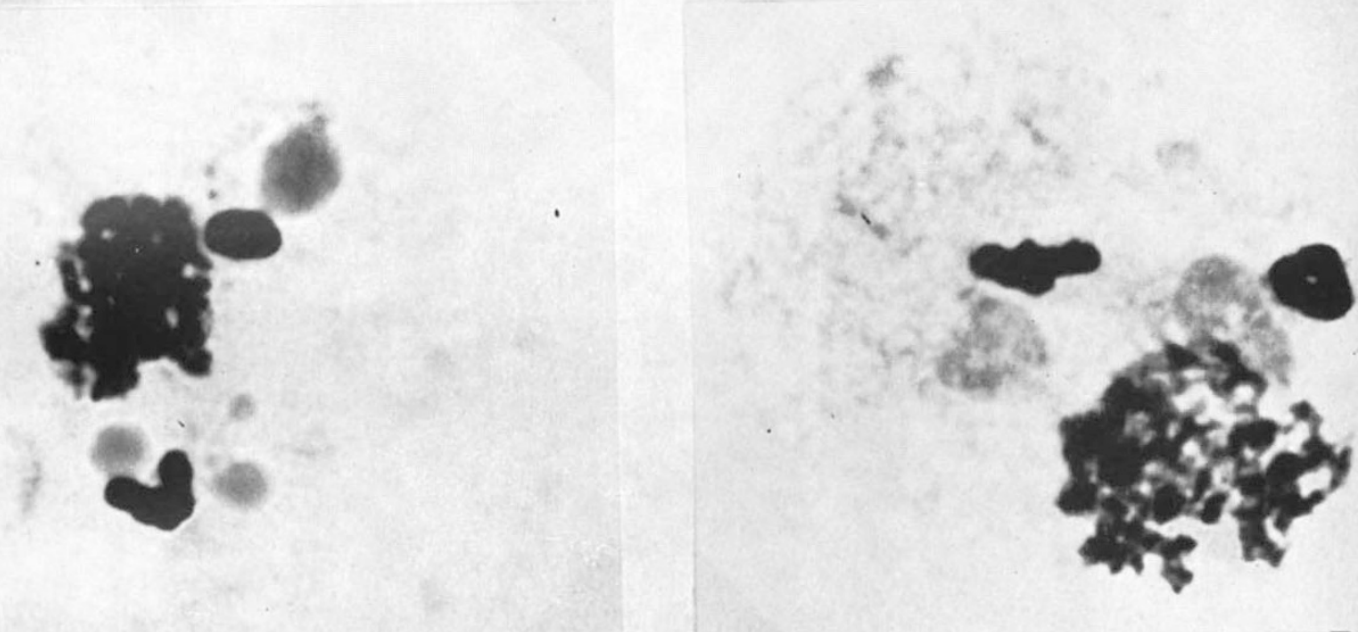

7

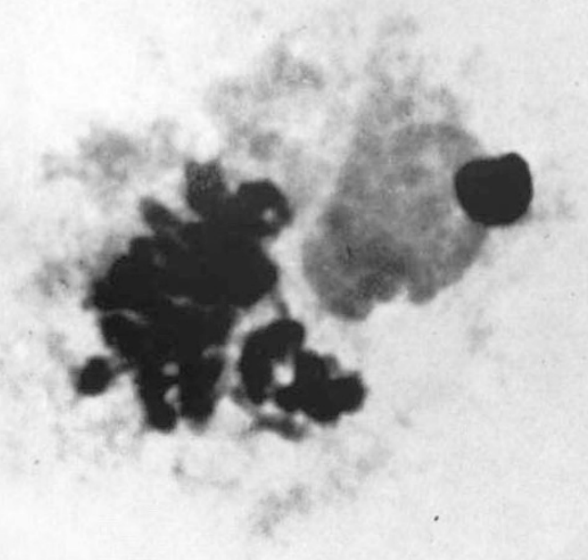

II 
retarded. Thus the normal and retarded chromosomes in a nucleus show a competition effect.

3. When all 7 bivalents form nucleoli they undergo normal condensation, and are able to organise a spindle but their centromeres fail to co-orientate. They show auto-orientation as in mitotic chromosomes or a lack of any definite orientation. In cells with mixed nuclei, the retarded bivalents also show failure of co-orientation but appear to be more efficient than the normal ones in their congression on the metaphase plate.

4. Thus dispersal of the nucleolus leads to two parallel systems of development in the same nucleus : nucleolar bivalents which are normal except for the centromere and non-nucleolar ones which are abnormal in their DNA-conditioned development.

5. Normal nucleoli might be regarded as available for metabolism of all chromosomes and dispersed nucleoli as available only for those to which they are attached. Or, perhaps preferably, chromosomes forming dispersed nucleoli might be capable of normal DNA-conditioned development while others are not. This leads to the suggestion that the capacities for nucleolar and DNA synthesis in a chromosome are correlated.

Acknowledgments. - I am greatly indebted to Prof. P. T. Thomas for suggesting the experiments and for advice and helpful criticism during the course of this work. My thanks are also due to Prof. E. T. Jones and Mr A. R. Beddows for providing the experimental material and for their kind encouragement. The award of one of their Science Research Scholarships by the Royal Commissioners for the Exhibition of 1851 , which enabled me to undertake this work, is gratefully acknowledged.

Note.-The observations described and discussed in this paper were submitted to the University of Wales as part of a Ph.D. thesis.

\section{REFERENCES}

ALFERT, MAX. 1954. Composition and structure of giant chromosomes. Int. Rev. Cytology, 3, $13 \mathrm{I}-\mathrm{I} 64$.

ANSLEY, H. R. I954. A cytochemical and cytophotometric study of alternative pathways of meiosis in the House Centipede. Chromosoma, 6, 656-695.

BRACHET, J. 1947. Nucleic acid in the cell and embryo. Symp. S.E.B., x, 207-224. CLEVELAND, L. R. I949. The whole life cycle of chromosomes and their coiling systems. Trans. Amer. Phil. Soc., 39 (1), I-1oo.

Darlington, G. D. 1937. Recent Advances in Cytology, 2nd Ed. London : Churchill.

DARlington, G. D. 1947. Nucleic acid and the chromosomes. Symp. S.E.B., I, 252-269.

Darlington, C. D. 1955. The chromosome as a physico-chemical entity. Nature, I76, 4494 .

HeItz, E. I93I. Die Urzache der gesetzmassingen Zahl, Lage, Form und Pflanzlicher Nukleolen, Planta, 12 , 775-844.

JACOBSON, W., AND WEBB, M. I952. The two types of nucleo proteins during mitosis. Expl. Cell. Res., $3,163$.

JACOBSON, w., AND WEBb, M. I952a. Nucleoproteins and cell division. Endeavour, 2, 200-207.

MCCLINTOCK, B. I934. The relation of a particular chromosomal element to the development of the nucleoli in Zea Mays. Zeitz, Zelef, U. Miker, Anat., 2I, 194-328. 
MIRSKY, A. E. I95I. Some chemical aspects of the cell nucleus. Genetics in the 2oth Century, pp. 127-I54.

MIRSKy, A. E., AND RIS, H. I95I. Cited by Alfert. Int. Rev. Cytology, 3, I3I-I 76.

POLlister, A. E. 1952. Nucleoproteins of the nucleus. Expt. Cell. Res. Suppl. 2, pp. 59-74.

RICH, A., AND Watson, J. D. I954. Some relations between DNA and RNA. Proc. Nat. Acad. Sc., $40,759-764$.

RIS, H., AND MIRSKY, A. E. I 949 . The state of chromosomes in the interphase nucleus. 7. Gen. Physiol., 32, 489-502.

schultz, J. 1947. The nature of heterochromatin. Symp. Quant. Biol., 12, 179-191 . sWIFT, H. 1953. Quantitative aspects of nucleoproteins. Int. Rev. Cytology, 2, I-69 WALKER, P. M. B., AND YATES, H. B. I952. Nuclear components of dividing cells. Proc. Roy. Soc. (London), B. 140, 274-299.

White, M. J. D. I95I. Nucleus, chromosomes and genes. Cytology and Cell Physiology, 2nd Ed. pp. 183-230. Oxford University Press.

Wilson, G. B., AND coleman, P. G. - 1952. The ontogeny of chromosome and chromonema spirals. Cytologia, Tokyo, 17, 27-28. 